\title{
INTEGRAL REPRESENTATIONS OF INVARIANT MEASURES
}

BY

\author{
ASHOK MAITRA
}

\begin{abstract}
In this paper we prove, under suitable conditions, several representation theorems for invariant measures arising out of the action of a family of measurable transformations $\mathcal{T}$ on a measurable space $(X, \mathcal{Q})$. Our results unify and extend results of Farrell and Varadarajan on the representation of invariant measures.
\end{abstract}

1. Introduction. There is considerable literature on the problem of representing invariant measures as mixtures or integral averages of ergodic measures. One of the earliest contributions to this field was made by Kryloff and Bogoliouboff [9], who proved the representability of invariant measures arising out of the action of a group of homeomorphisms on a compact metric space. Subsequently, several authors, notably Farrell [4] and Varadarajan [16], extended the result of Kryloff and Bogoliouboff to more general situations. (For a rather extensive bibliography on this subject, the reader is referred to [12] and [16].)

We deal in this paper with the representation problem mentioned above. The point of departure of our efforts is the treatment of this problem by Farrell [4]. The situations considered by Farrell may be loosely described as follows. $X$ is a Polish space, or more generally, an analytic subset of a Polish space, $\mathcal{Q}$ is the Borel $\sigma$-field of $X$ and $\mathcal{T}$ is a nonempty family of Borel measurable transformations on $X$ into $X$. Under suitable restrictions on $\mathcal{T}$ of a topological and group-theoretic kind, Farrell proves the representability of invariant measures. The aim of this article is to unify these results and to extend them to the case where $X$ is a universally measurable separable metric space. This is done by eliminating the topological ingredients of Farrell's treatment and replacing them by conditions of a purely measure-theoretic kind.

It should be mentioned that in one respect our methods differ from those of Farrell. In checking that there are enough extreme points in the convex set of

Received by the editors October 4, 1975.

AMS (MOS) subject classifications (1970). Primary 28A65, 28A70; Secondary 47A35.

Key words and phrases. Measurable transformations, invariant measures, extreme points, sufficient $\sigma$-fields, representability. 
invariant measures for representing invariant measures, Farrell (and also Varadarajan) has to imbed the given system $(X, \mathcal{Q}, \mathcal{T})$ Borel isomorphically into a system consisting of transformations acting continuously on a compact metric space (a precise formulation of this is Theorem 3.2 in [16]) and then appeal to the Krein-Milman theorem. But, as remarked by Varadarajan [16, p. 199], such an imbedding is not possible when the basic space is not analytic. We get around this difficulty by using regular conditional probabilities given a suitable countably generated sub- $\sigma$-field of the almost invariant $\sigma$-field; the regular conditional probabilities provide us with all the extreme points needed for representing invariant measures. However, like Farrell, we too exploit the sufficiency of the almost invariant $\sigma$-field for the family of invariant measures in all the situations where we are able to establish the representability of invariant measures. On the whole our methods seem to be simpler and more direct than those of Farrell.

The paper is organized as follows. $\$ 2$ introduces the basic definitions and notation. In $\$ 3$ we record some preliminary results which are needed in the proofs of the representation theorems. $\$ 4$ contains the main representation theorem and some of its consequences. In $\$ 5$ representation theorems are proved for nonseparable families of measurable transformations. In $\$ 6$ we give examples which help elucidate various aspects of the theory.

2. Basic definitions and notation. Let $(X, \mathbb{Q})$ be a measurable space. A transformation $T: X \rightarrow X$ is said to be measurable if for each $A \in \mathbb{Q}, T^{-1}(A)$ $\in \mathbb{Q}$. Let $\mathcal{T}$ be a nonempty family of measurable transformations on $X$. A measure will always mean a countably additive probability measure. A measure $\mu$ on $\mathbb{Q}$ is said to be invariant with respect to $\mathcal{T}$ if for each $A \in \mathbb{Q}$ and $T \in \mathcal{T}, \mu(A)=\mu\left(T^{-1}(A)\right)$. We denote the set of all measures invariant with respect to $\mathscr{T}$ by $\mathscr{P}_{\mathscr{g}}$. Plainly, $\mathscr{P}_{\mathscr{g}}$ is a convex set and the set of extreme points of $\mathscr{P}_{\text {g }}$ is denoted by ex $\mathcal{P}_{\mathscr{\sigma}}$. A set $E \in \mathbb{Q}$ is said to be null with respect to $\mathcal{T}$ if $\mu(E)=0$ for every $\mu \in \mathscr{P}_{\text {g }}$. The $\sigma$-ideal of null sets is denoted by $\Re_{\mathscr{G}}$. A set $E \in \mathbb{Q}$ is invariant for $\mathcal{G}$ if $E=T^{-1}(E)$ for every $T \in \mathcal{T}$. If $\mu \in \mathscr{P}_{\mathscr{\sigma}}$, we say that $E$ is $\mu$-almost invariant if $\mu\left(E \Delta T^{-1}(E)\right)=0$ for every $T \in \mathscr{T}$. Say that $E \in \mathbb{Q}$ is almost invariant for $\mathcal{G}$ if $E$ is $\mu$-almost invariant for every $\mu \in \mathscr{P}_{\mathscr{T}}$, i.e., if $E \triangle T^{-1}(E) \in \mathcal{N}_{\mathscr{J}}$ for every $T \in \mathcal{T}$. The collection of invariant, $\mu$-almost invariant and amost invariant sets are denoted by $I_{\sigma}$, $g_{\sigma}^{\mu}$ and $\bar{q}_{\sigma}$, respectively. These collections are easily seen to be $\sigma$-fields and will be called, respectively, the invariant, $\mu$-almost invariant and almost invariant $\sigma$ fields. A measure $\mu \in \mathcal{P}_{\mathscr{T}}$ is said to be ergodic if the restriction $\mu \mid \bar{\Phi}_{\mathscr{T}}$ of $\mu$ to $\bar{\Phi}_{\mathscr{T}}$ is $0-1$ valued. [Another definition of an ergodic measure would be to require that the invariant measure be $0-1$ valued on the invariant $\sigma$-field $\Phi_{\sigma \cdot}$. In general, this definition is less stringent than the one we have adopted, as Example 1 in $\S 6$ shows. In case $g_{\text {ब }}$ is sufficient for $\mathscr{P}_{\mathscr{T}}$, the two definitions coincide (see 
Lemma 5).] Denote the set of ergodic measures by $\rho_{\mathscr{g}}^{e}$. Say that the family $\mathcal{T}$ is separable if there is a countable set $\mathcal{T}_{0} \subseteq \mathcal{T}$ such that whenever a measure is invariant with respect to $\mathscr{T}_{0}$, it is invariant with respect to $\mathcal{T}_{\text {, i.e., }} \mathscr{P}_{\mathscr{T}}=\mathscr{P}_{\mathscr{T}_{0}}$. The notion of separability introduced above will help us in formulating a general representation theorem for invariant measures which subsumes several known results.

In order to define the representability of invariant measures, we equip the set ex $\mathscr{P}_{\mathscr{\sigma}}$ with a $\sigma$-field $\Sigma_{\mathscr{\sigma}}: \Sigma_{\mathscr{\sigma}}$ is the smallest $\sigma$-field of subsets of ex $\mathscr{P}_{\mathscr{\sigma}}$ which makes the maps $\mu \rightarrow \mu(E), E \in \mathbb{Q}$, measurable. If $\mu \in \mathscr{P}_{\mathscr{\sigma}}$, say that $\mu$ is representable if there is a measure $m$ on $\Sigma_{\mathscr{\sigma}}$ such that $\mu(E)=\int_{\text {ex } \Phi_{\mathscr{g}}} \nu(E) d m(\nu)$ for every $E \in \mathbb{Q}$. In this case $m$ is called a representing measure for $\mu$.

We say that $\mathcal{T}$ is a group acting on $(X, \mathbb{Q})$ if $\mathcal{T}$ is a nonempty family of measurable transformations on $X$ to $X$ such that (a) $\mathcal{\sigma}$ is a group under composition, and (b) the identity transformation on $X$ is the identity of the group $\mathcal{T}$. Say that $\mathcal{T}$ is a group acting measurably on $(X, \mathbb{Q})$ if $\mathcal{G}$ is a group acting on $(X, \mathbb{Q})$ such that $\mathcal{T}$ is a topological group and the mapping $(T, x)$ $\rightarrow T(x)$ is $(\mathscr{B} \times \mathbb{Q}, \mathbb{Q})$-measurable, where $B$ is the Baire $\sigma$-field of $\mathscr{T}$. Recall that the Baire $\sigma$-field of $\mathcal{G}$ is the $\sigma$-field generated by the zero sets of $\mathscr{T}$. In case $\mathcal{T}$ is metrizable, the Baire $\sigma$-field is just the Borel $\sigma$-field; and if $\sigma$ is compact, the Baire $\sigma$-field is the $\sigma$-field generated by compact $G_{\delta}$ sets. Analogously, one defines the notion of a semigroup acting on $(X, \mathbb{Q})$.

A measure $\mu$ on a measurable space $(X, \mathbb{Q})$ is perfect if for every $\mathbb{Q}$ measurable function $f$ into the reals, there is a Borel subset $B$ of the real line such that $B \subseteq f(X)$ and $\mu\left(f^{-1}(B)\right)=1$. A measurable space $(X, \mathbb{Q})$ is said to be a perfect space if (i) $\mathbb{Q}$ contains singletons, (ii) $\mathbb{Q}$ is countably generated, and (iii) every measure on $\mathbb{Q}$ is perfect. A separable metric space $X$ is said to be universally measurable if $X$ is measurable with respect to every measure on the Borel subsets of the completion of $X$, or equivalently, if $X$ is measurable with respect to every measure on the Borel subsets of any Polish space in which $X$ can be homeomorphically imbedded. It is known that if $X$ is a universally measurable separable metric space and $\mathbb{Q}$ is the Borel $\sigma$-field of $X$, then $(X, \mathbb{Q})$ is a perfect space; and, conversely, if $(X, \mathbb{Q})$ is a perfect space, then $X$ can be metrized so that $X$ is a universally measurable separable metric space and $Q$ is its Borel $\sigma$-field. Our results can therefore be formulated either in terms of perfect spaces or universally measurable separable metric spaces. We have chosen the former for our main results because of its measure-theoretic flavour. Marczewski [11] and Ryll-Nardzewski [15] are good references for information on perfect measures and related matters.

Let $\mathcal{P}$ be a family of measures on a measurable space $(X, \mathbb{Q})$ and let $\mathcal{C}$ be a sub- $\sigma$-field of $\mathcal{Q}$. Say that $\mathcal{C}$ is sufficient for $\mathcal{P}$ if for every $E \in \mathbb{Q}$, there is a realvalued $\mathcal{C}$-measurable function $f$ on $X$ such that $\mu(A \cap E)=\int_{A} f d \mu$ for every 
$A \in \mathcal{C}$ and $\mu \in \mathcal{P}$. We say that $\mathcal{C}$ is separating for $\mathcal{P}$ if $\mu_{1}, \mu_{2} \in \mathcal{P}$ and $\mu_{1} \mid \mathcal{C}$ $=\mu_{2} \mid \mathcal{C}$ imply $\mu_{1}=\mu_{2}$. It is easy to see that if $\mathcal{C}$ is sufficient for $\mathscr{P}$, then $\mathcal{C}$ is separating for $\Phi$.

3. Auxiliary results. Throughout this $\operatorname{section}(X, \mathbb{Q})$ will be a fixed measurable space and $\mathcal{T}$ a nonempty family of measurable transformations on $X$ to $X$. There will be no retrictions on the measurable space $(X, \mathbb{Q})$ and any assumptions made regarding $\mathcal{T}$ will be explicitly stated.

Leman 1. Suppose that $\bar{\Phi}_{\mathscr{\sigma}}$ is sufficient for $\mathscr{P}_{\text {g }}$. Then

(i) $\operatorname{ex} \mathscr{P}_{\mathscr{g}}=\mathscr{P}_{\mathscr{g}}^{e}$,

(ii) the following conditions are equivalent:

(a) every $\mu \in \Phi_{\sigma}$ is representable and has a unique representing measure,

(b) for every $A \in \bar{\Phi}_{\text {G }}$ and $\mu \in \mathscr{P}_{\text {ब }}$ such that $\mu(A)>0$, there is $\nu$ $\in \operatorname{ex} \mathscr{P}_{\text {g }}$ such that $\nu(A)=1$.

The above result is due to Farrell and the reader is referred to [4] for a proof. It should be noted that ex $\mathcal{P}_{\text {g }} \subseteq \Phi_{\text {g }}^{e}$ always (cf., for example, [4, Lemma 1]); Example 4 in $\$ 6$ shows that the reverse inclusion is, in general, false. In all the situations where we are able to prove the representability of invariant measures, it turns out that the almost invariant $\sigma$-field is sufficient for $\mathscr{P}_{\text {g }}$ so that the ergodic measures are just the extreme points of $\Phi_{\text {g }}$. (Warning. We are not claiming here that a necessary condition for the representability of invariant measures is the sufficiency of $\overline{\mathscr{G}}_{\mathrm{g}}$ for $\mathscr{P}_{\mathrm{g}}$; indeed, it is not, as Example 5 shows.) Jacob Feldman has proved the following measure-theoretic charac-

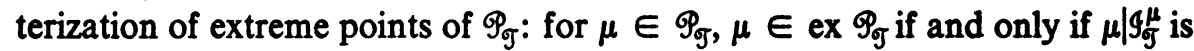
$0-1$ valued. The details are to be found in $[13, \S 10]$. We shall have no occasion to use this result.

Lemma 1(ii) extends a result of Blum and Hanson [2], who proved the equivalence of conditions (a) and (b) when $\sigma$ consists of a single bijective and bimeasurable transformation of $X$ onto $X$. Plainly, condition (a) always implies condition (b); in general, the reverse implication is false, as Example 3 shows.

We now investigate conditions under which the almost invariant $\sigma$-field is sufficient for $\Phi_{\text {g }}$. We begin with a result of Jacob Feldman. See $[13,810]$ for a proof.

LeMma 2. Suppose $\mu, \lambda$ are measures on $(X, \mathbb{Q})$ such that $\mu \in \mathscr{P}_{\mathscr{g}}$ and $\lambda$ is absolutely continuous with respect to $\mu$. Let $f=d \lambda \mid d \mu$. Then $\lambda \in \Phi_{\text {g }}$ if and only if $f \circ T=f$ a.e. $[\mu]$ for every $T \in \mathcal{T}$.

LEMMA 3. If $\mathcal{T}_{0}$ is a subfamily of $\mathcal{T}_{\text {such }}$ that $\mathscr{\Phi}_{\mathscr{T}_{0}}=\mathscr{\Phi}_{\mathscr{\sigma}}$, then $\bar{\Phi}_{g}=\bar{\Phi}_{\Phi_{0}}$. 


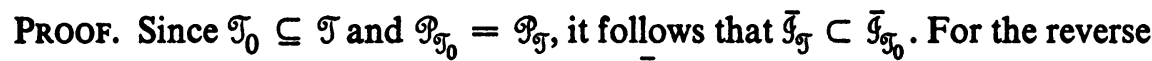
inclusion, let $h$ be a bounded, nonnegative $\bar{\Phi}_{\sigma_{0}}$-measurable function on $X$. Fix $\mu \in \mathscr{P}_{\mathscr{T}}=\mathscr{P}_{\mathscr{T}_{0}}$. If $\int h d \mu=0$, then $\int h \circ T d \mu=0$ for every $T \in \mathcal{T}$, so that $h \circ T=h$ a.e. $[\mu]$ for every $T \in$ T. Suppose now that $\int h d \mu=\alpha>0$. Define a measure $\lambda$ on $\mathbb{Q}$ by: $\lambda(E)=\alpha^{-1} \int_{E} h d \mu, E \in \mathbb{Q}$. As $h$ is $\bar{\Phi}_{\sigma_{0}}$-measurable, $h \circ T=h$ a.e. $[\mu]$ for every $T \in \mathscr{T}_{0}$. Hence, by the if part of Lemma 2 , $\lambda \in \mathscr{P}_{\mathscr{T}_{0}}$ and so $\lambda \in \mathscr{P}_{\mathscr{F}}$. It follows now from the 'only if' part of Lemma 2 that $h \circ T=h$ a.e. $[\mu]$ for every $T \in \mathcal{T}$. Thus, in any case, $h \circ T=h$ a.e. $[\mu]$ for every $T \in \mathcal{T}$ and any $\mu \in \mathscr{P}_{\mathscr{g}}$. Consequently, $h$ is $\overline{\mathscr{G}}_{\mathscr{\sigma}}$-measurable, which completes the proof.

LEMMA 4. If $\mathcal{T}$ is separable, then $\bar{\Phi}_{\mathscr{\sigma}}$ is sufficient for $\mathscr{P}_{\mathcal{G}}$.

Proof. Let $\mathcal{T}_{0}$ be a countable subfamily of $\mathcal{T}$ such that $\mathscr{P}_{\mathscr{F}_{0}}=\mathscr{P}_{\mathscr{\sigma}}$. As $\mathcal{T}_{0}$ is countable, it follows by a result of Farrell [4, Theorem 2] that $\bar{g}_{\sigma_{0}}$ is sufficient for $\mathscr{P}_{\sigma_{0}}=\mathscr{P}_{\sigma}$. The present proof is completed by appealing to Lemma 3 .

One may wonder if the separability of $\mathcal{T}$ implies the sufficiency of the invariant $\sigma$-field $g_{g}$ for $\mathcal{P}_{g}$. This is not so. Indeed, Farrell has an example of a semigroup, consisting of two elements and acting on a measurable space such that the invariant $\sigma$-field is not sufficient for the family of invariant measures. See [4, p. 452]. However, Farrell has also shown that for a countable group acting on a measurable space the invariant $\sigma$-field is sufficient [4, Theorem 3]. Example 1 shows that this result cannot be extended to separable groups acting measurably on a measurable space.

The sufficiency of $\mathcal{G}_{\mathscr{\sigma}}$ for $\mathscr{P}_{\mathscr{T}}$ does imply the sufficiency of $\bar{g}_{\mathscr{G}}$. More precisely, we have

LEMMA 5. If $\mathcal{G}_{\mathcal{\sigma}}$ is sufficient for $\mathcal{P}_{\sigma}$, then $\bar{\Phi}_{\sigma}=\sigma\left(\mathcal{G}_{\sigma} \cup \vartheta_{\sigma}\right)$, where, for any family $\mathcal{E}$ of subsets of $X, \sigma(\mathcal{E})$ is the $\sigma$-field generated by $\mathcal{E}$. Consequently, if $\mathcal{G}_{\mathcal{G}}$ is sufficient for $\mathcal{P}_{\text {g }}$, then so is $\bar{\Phi}_{\text {g }}$.

Proof. Plainly, $\sigma\left(\mathcal{I}_{\sigma} \cup \Re_{\sigma}\right) \subseteq \bar{g}_{\text {g }}$. For the reverse inclusion, let $h$ be a

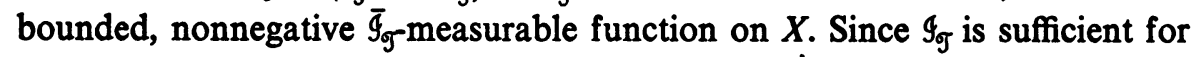
$\mathscr{P}_{\mathscr{\sigma}}$, there is a nonnegative $\Im_{\mathscr{\sigma}}$-measurable function $h^{*}$ on $X$ such that

$$
\int_{A} h d \mu=\int_{A} h^{*} d \mu
$$

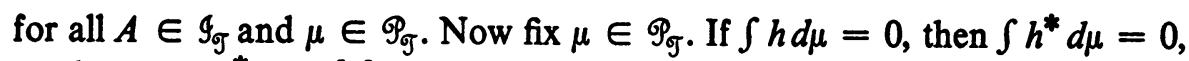
so that $h=h^{*}$ a.e. $[\mu]$. Suppose, then, that $\int h d \mu=\alpha>0$. Define $\lambda_{1}(E)$

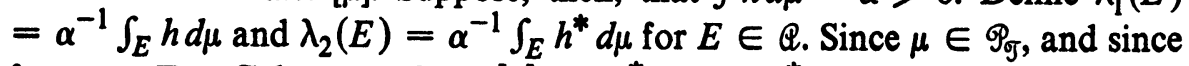
for every $T \in \mathcal{J}, h \circ T=h$ a.e. [ $\mu$ ] and $h^{*} \circ T=h^{*}$, it follows by the 'if' part of Lemma 2 that $\lambda_{1}, \lambda_{2} \in \Phi_{\text {F }}$. But from (1) we have $\lambda_{1}=\lambda_{2}$ on $g_{\text {F. }}$. As $g_{\sigma}$ is separating for $\mathscr{P}_{\mathscr{\sigma}}$, it follows that $\lambda_{1}=\lambda_{2}$ on $\&$ Consequently, $h=h^{*}$ a.e. $[\mu]$. Thus, in any case, $h=h^{*}$ a.e. $[\mu]$ for all $\mu \in \mathscr{P}_{\sigma}$. Hence $h$ is $\sigma\left(I_{\sigma} \cup \mathscr{T}_{\sigma}\right)$ - 
measurable, which completes the proof of the first assertion of the lemma. The second assertion follows trivially from the first.

Problems similar to those considered in Lemmas 3, 4 and 5 are discussed in Basu [1]. However, for Basu, the relevant family of measures is most often a proper subfamily of the family of invariant measures. We do not know if Lemma 5 holds in Basu's setup.

4. Main representation theorem. Towards the proof of the main representation theorem we first establish two lemmas. These lemmas essentially show that regular conditional probabilities give us enough ergodic measures for representing invariant measures.

For the lemmas, we assume that $\mathbb{Q}$ is a countably generated $\sigma$-field on $X, \mathcal{T}$ is a separable family of measurable transformations on $(X, \mathbb{Q})$ and $\mu$ is a fixed measure in $\mathscr{P}_{\sigma}$. Further, let $\mathcal{C}$ be a countably generated sub- $\sigma$-field of $\bar{g}_{\sigma}$ and let $Q(x, E)$ be a $\mu$-regular conditional probability on $Q$ given $C$, i.e., (i) for fixed $E \in \mathbb{Q}, Q(\cdot, E)$ is a $\mathcal{C}$-measurable function on $X$, (ii) for fixed $x \in X, Q(x, \cdot)$ is a measure on $\mathbb{Q}$, and (iii) for every $E \in \mathbb{Q}$ and $A \in \mathcal{C}$,

$$
\mu(A \cap E)=\int_{A} Q(x, E) d \mu(x) .
$$

LEMMA 6. Under the above conditions, there is a set $X_{1} \in \mathcal{C}$ such that $\mu\left(X_{1}\right)=1$ and $Q(x, \cdot) \in \mathscr{P}_{\mathscr{\sigma}}$ for every $x \in X_{1}$.

Proof. Let $\mathcal{Q}_{0}$ be a countable field which generates $\mathbb{Q}$, and let $\mathcal{T}_{0}$ be a countable subfamily of $\mathcal{T}$ such that $\mathscr{P}_{\mathscr{T}}=\mathscr{P}_{\mathscr{T}_{0}}$. For $E \in \mathbb{Q}, A \in \mathcal{C}$ and $T \in \mathcal{T}$, we have

$$
\begin{aligned}
\mu(A \cap E) & =\int_{A} Q(x, E) d \mu(x), \text { and } \\
\mu\left(A \cap T^{-1}(E)\right) & =\int_{A} Q\left(x, T^{-1}(E)\right) d \mu(x) .
\end{aligned}
$$

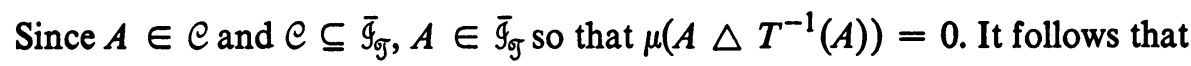

$$
\mu(A \cap E)=\mu\left(T^{-1}(A) \cap T^{-1}(E)\right)=\mu\left(A \cap T^{-1}(E)\right) .
$$

Consequently,

$$
\int_{A} Q(x, E) d \mu(x)=\int_{A} Q\left(x, T^{-1}(E)\right) d \mu(x) .
$$

This last equality holds for every $A \in \mathcal{C}$. It follows that, if $N_{E, T}=\{x$ $\left.\in X: Q(x, E) \neq Q\left(x, T^{-1}(E)\right)\right\}$, then $N_{E, T} \in \mathcal{C}$ and $\mu\left(N_{E, T}\right)=0$.

Set $X_{1}=X-U_{T \in \mathscr{T}_{0}} U_{E \in \mathbb{Q}_{0}} N_{E, T}$. Recalling that $\mathcal{T}_{0}$ and $\mathscr{Q}_{0}$ are countable, we see that $X_{1} \in \mathcal{C}$ and $\mu\left(X_{1}\right)=1$. We now check that $X_{1}$ works. So let $x$ 
$\in X_{1}$ and $T \in \mathcal{T}_{0}$. If $\lambda=Q(x, \cdot)$, then, clearly, $\lambda=\lambda T^{-1}$ on $\mathbb{Q}_{0}$ and, hence,

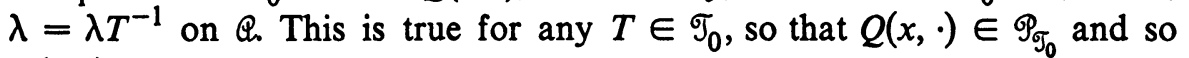
$Q(x, \cdot) \in \mathscr{P}_{\mathscr{g}}$. This completes the proof.

Lemma 7. Under the same conditions as in Lemma 6, there is a set $X_{2} \in \mathcal{C}$ such that (i) $\mu\left(X_{2}\right)=1$, (ii) for each $x \in X_{2}$ and $A \in \mathcal{C}, Q(x, A)=I_{A}(x)$, where $I_{A}$ is the indicator of the set $A$, and (iii) $Q(x, \cdot) \in \mathscr{P}_{\text {F }}$ for each $x \in X_{2}$.

Proof. It is well known that, since $\mathcal{C}$ is countably generated and $Q(x, E)$ is a regular conditional probability given $C$, there is a set $N \in \mathcal{C}$ such that $\mu(N)=0$ and $Q(x, A)=I_{A}(x)$ for each $x \in X-N$ and $A \in \mathcal{C}$; see, for example, [6, p. 352]. By Lemma 6, there is a set $X_{1} \in \mathcal{C}$ such that $\mu\left(X_{1}\right)$ $=1$ and $Q(x, \cdot) \in \mathcal{P}_{\mathscr{T}}$ for every $x \in X_{1}$. Let $X_{2}=X_{1}-N$, so that $X_{2} \in \mathcal{C}$. It is now clear that $X_{2}$ satisfies the requirements of the present lemma, which completes the proof.

Our main result on the representability of invariant measures is

THEOREM 1. Let $(X, \mathbb{Q})$ be a perfect space and let $\mathcal{T}$ be a separable family of measurable transformations on $X$. Then every invariant measure is representable and has a unique representing measure. Moreover, $\left(\mathrm{ex} \mathscr{P}_{\mathscr{\sigma}}, \Sigma_{\mathscr{\sigma}}\right)$ is a perfect space.

Proof. Since $\sigma$ is separable, it follows from Lemma 4 that $\bar{q}_{\sigma \text { is sufficient for }}$ $\mathscr{P}_{\sigma}$. Consequently, as $\mathbb{Q}$ is countably generated, there is a countably generated $\sigma$-field $e$ such that $\bar{\Phi}_{\mathscr{T}}=\sigma\left(e \cup \Re_{\sigma}\right)[3$, Theorem 1].

Now fix $\mu \in \mathscr{P}_{\mathcal{F}}$. As $\mu$ is perfect, there is, according to a result in Jirina [8], a $\mu$-regular conditional probability $Q(x, E)$ on $\mathbb{Q}$ given $\mathcal{C}$ Note that Lemma 7 is now applicable. Fix a set $X_{2} \in \mathcal{C}$ satisfying the conditions of Lemma 7.

We now claim that $Q(x, \cdot) \in$ ex $\mathscr{P}_{\text {f }}$ for every $x \in X_{2}$. To see this, let $x \in X_{2}$. Then conditions (ii) and (iii) of Lemma 7 imply that $Q(x, \cdot)$ $\in \mathscr{P}_{\text {g }}$ and $Q(x, \cdot) \mid \mathcal{C}$ is $0-1$ valued. Since $\overline{\mathscr{G}}_{\mathscr{\sigma}}=\sigma\left(\mathcal{C} \cup \Re_{\sigma}\right)$ and $Q(x, \cdot) \in \mathscr{P}_{\mathscr{T}}$, it follows that $Q(x, \cdot) \mid \bar{g}_{\mathscr{G}}$ is $0-1$ valued, so that $Q(x, \cdot) \in \mathcal{P}_{\mathscr{g}}^{e}$. As $\bar{g}_{\mathscr{G}}$ is sufficient for $\mathscr{P}_{\text {F }}$, Lemma 1(i) now implies that $Q(x, \cdot) \in$ ex $\mathscr{P}_{\text {g }}$.

The discussion in the preceding paragraph enables us to define a map $\varphi: X_{2} \rightarrow$ ex $\mathscr{P}_{\mathscr{T}}$ by $\varphi(x)=Q(x, \cdot)$. We first study some properties of this map. Clearly $\varphi$ is $\left(e \cap X_{2}, \Sigma_{\mathscr{\sigma}}\right)$-measurable, where $e \cap X_{2}$ is the trace of $e$ on $X_{2}$. On the other hand, if $A \in \mathcal{C} \cap X_{2}$ then $\varphi(A)=\left\{\nu \in \operatorname{ex} \mathscr{P}_{\mathscr{g}}: \nu(A)=1\right\}$. To see this, let $A \in \mathcal{C} \cap X_{2}$. Since $X_{2} \in \mathcal{C}$, it follows that $A \in \mathcal{C}$. By condition (ii) of Lemma 7, $x \in X_{2}$ implies $Q(x, A)=I_{A}(x)$. Hence, if $\nu \in \varphi(A)$, then $\nu$ $\in$ ex $\mathscr{P}_{\text {g }}$ and $\nu(A)=1$. Conversely, suppose $\nu \in \operatorname{ex} \mathscr{P}_{\text {F }}$ and $\nu(A)=1$. So $\nu \mid e_{\text {is }}$ $0-1$ valued, and since $C$ is countably generated, $\nu$ concentrates on a $C$-atom, i.e., there is a $C_{\text {atom, say }} B$, such that $\nu(B)=1$. Plainly, $B \subseteq A$. Let $x \in B$. Since $A \subseteq X_{2}$, it follows that $x \in X_{2}$ and so condition (ii) of Lemma 7 implies that

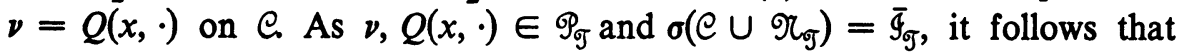


$\nu=Q(x, \cdot)$ on $\bar{g}_{\sigma}$. But $\bar{\Phi}_{\mathscr{T}}$ is separating for $\mathscr{\Phi}_{\mathscr{T}}$, so $\nu=Q(x, \cdot)$ on $\mathbb{Q}$, hence $\nu=\varphi(x) \in \varphi(A)$. Consequently, if $A \in \mathcal{C} \cap X_{2}$ then $\varphi(A) \in \Sigma_{\text {g }}$.

To prove the representability of $\mu$, let $E \in \mathbb{Q}$. We then have

$$
\begin{aligned}
\mu(E) & =\int_{X} Q(x, E) d \mu(x) \\
& =\int_{X_{2}} Q(x, E) d \mu(x) \quad\left(\text { since } \mu\left(X_{2}\right)=1\right) \\
& =\int_{\operatorname{ex}_{9}} \nu(E) d m(\nu) \quad \text { (change of variable theorem) }
\end{aligned}
$$

where $m=\mu \varphi^{-1}$. This holds for any $E \in \mathbb{Q}$ and so $\mu$ is representable.

Next we show that $\mu$ has at most one representing measure. Let, then, $n$ be a representing measure for $\mu$. Suppose $A \in \mathcal{C} \cap X_{2}$. We have already proved that $\varphi(A)=\left\{\nu \in \operatorname{ex} \mathscr{P}_{g}: \nu(A)=1\right\}$ and that $\varphi(A) \in \Sigma_{g}$. We now compute $n(\varphi(A))$ as follows:

$$
\begin{aligned}
n(\varphi(A)) & =n\left(\left\{\nu \in \operatorname{ex} \mathscr{P}_{\mathscr{\sigma}}: \nu(A)=1\right\}\right) \\
& =\int_{\left\{\nu \in \operatorname{ex} \Phi_{g}: \nu(A)=1\right\}} \nu(A) d n(\nu) \\
& =\int_{\operatorname{ex} \Phi_{g}} \nu(A) d n(\nu) \quad\left(\text { since, for every } \nu \in \operatorname{ex} \mathscr{P}_{\sigma}, \nu(A)=0 \text { or } 1\right) \\
& =\mu(A) .
\end{aligned}
$$

In particular, $n\left(\varphi\left(X_{2}\right)\right)=\mu\left(X_{2}\right)=1$. Now let $H \in \Sigma_{\sigma}$. Then $\varphi^{-1}(H) \in \mathcal{C}$ $\cap X_{2}$ and $\varphi\left(\varphi^{-1}(H)\right)=H \cap \varphi\left(X_{2}\right)$. Furthermore,

$$
\mu\left(\varphi^{-1}(H)\right)=n\left(\varphi\left(\varphi^{-1}(H)\right)\right)=n\left(H \cap \varphi\left(X_{2}\right)\right)=n(H) .
$$

The last equality follows from the fact that $n\left(\varphi\left(X_{2}\right)\right)=1$. As $H$ is an arbitrary set in $\Sigma_{\mathscr{\sigma}}$, we have therefore proved that $n=\mu \varphi^{-1}$. From this it follows that any two representing measures for $\mu$ must be identical.

Let $m$ be the representing measure for $\mu$. We show that $m$ is perfect. Let, then, $f$ be a real-valued $\Sigma_{\mathscr{\sigma}}$-measurable function on ex $\mathscr{P}_{\sigma}$. So $f \circ \varphi$ is a realvalued $\mathrm{C} \cap X_{2}$-measurable function on $X_{2}$. Since $\mu$ is perfect, so is $\mu \mid \mathcal{C}$. From this and the facts that $X_{2} \in \mathcal{C}$ and $\mu\left(X_{2}\right)=1$, it follows that $\mu \mid \mathcal{C} \cap X_{2}$ is perfect. Hence there is a Borel subset $B$ of the real line such that $B$ $\subseteq f\left(\varphi\left(X_{2}\right)\right)$ and $\mu\left(\varphi^{-1}\left(f^{-1}(B)\right)\right)=1$. Since $m=\mu \varphi^{-1}$,

$$
m\left(f^{-1}(B)\right)=\mu\left(\varphi^{-1}\left(f^{-1}(B)\right)\right) .
$$

Thus $m\left(f^{-1}(B)\right)=1$ and $B \subseteq f\left(\right.$ ex $\left.\mathscr{P}_{\digamma}\right)$, which proves that $m$ is perfect. 
Since the map that sends each invariant measure to its representing measure sets up a one-one correspondence between invariant measures and measures on $\Sigma_{\mathscr{F}}$, the arguments of the previous paragraph show that every measure on $\Sigma_{\mathscr{\sigma}}$ is perfect. That $\boldsymbol{\Sigma}_{\mathscr{\sigma}}$ is countably generated and contains singletons follows by routine arguments from the fact that $Q$ is countably generated; see, for instance, [5]. We have thus established that $\left(\mathrm{ex} \mathscr{P}_{\widetilde{\sigma}}, \Sigma_{\sigma}\right)$ is a perfect space, which terminates the proof of Theorem 1.

REMARK 1. The proof of the existence and uniqueness of the representing measure for an invariant measure given above can be considerably shortened by invoking Lemma 1(ii). Since in Theorem $1 \overline{\mathscr{G}}_{\mathscr{F}}$ is sufficient for $\mathscr{P}_{\mathcal{F}}$, so, in view of Lemma 1(ii), it suffices to check condition (b) of Lemma 1(ii) in order to prove the existence and uniqueness of the representing measure. To do this, let $\mu \in \mathcal{P}_{\mathscr{\sigma}}, A \in \bar{g}_{\sigma}$ such that $\mu(A)>0$. With the same notation as in the proof above, we have $\mu\left(A \cap X_{2}\right)>0$ because $\mu\left(X_{2}\right)=1$. As $\bar{q}_{\text {g }}=\sigma\left(C \cup \vartheta_{g}\right)$, there is a set $A^{\prime} \in \mathcal{C}$ such that $A \triangle A^{\prime} \in \Re_{g}$, so that $\mu\left(A \triangle A^{\prime}\right)=0$. Hence $\mu\left(A^{\prime} \cap X_{2}\right)>0$. Thus $A^{\prime} \cap X_{2}$ is a nonempty set in $\mathcal{C}$. Let $x \in A^{\prime} \cap X_{2}$ and $\nu=Q(x, \cdot)$. Then $\nu \in \operatorname{ex} \mathscr{P}_{\sigma}$ and $\nu\left(A^{\prime}\right)=1$. As $A \Delta A^{\prime} \in \mathcal{\vartheta}_{g}$, we have $\nu(A)=1$. We have presented a longer proof to keep the exposition as self-contained as possible and also because it gives more insight into the representation problem.

REMARK 2. The condition in Theorem 1 requiring that every measure on $\mathbb{Q}$ be perfect (acutally, for the proof of Theorem 1, we only need that every invariant measure on $\mathscr{Q}$ be perfect) cannot be relaxed in general. Indeed, Varadarajan has given an example of a measurable space $(X, \mathbb{Q})$, where $X$ is a separable metric space and $Q$ is the Borel $\sigma$-field of $X$, and a countable group I acting on $(X, \mathbb{Q})$ such that there are uncountably many invariant measures but no ergodic measures, so that no invariant measure is representable; see $[16, p .217]$. On the other hand, dropping the condition that $\mathscr{T}$ is separable from Theorem 1 may lead to the same unpleasant consequences even when the space $(X, \mathbb{Q})$ is a standard Borel space, and so, a fortiori, a perfect space; see Example 2 in $\$ 6$.

REMARK 3. Theorem 1 constitutes a generalization of Theorem 5 in Farrell [4] in two directions: first, Farrell's assumption that the family of measurable transformations be countable is weakened, and second, the validity of Farrell's theorem is extended from Polish spaces to universally measurable separable metric spaces. Though the first direction of generalization is a trivial one, the second is not, for reasons mentioned in the Introduction. However, even the replacement of the condition that the family of transformations be countable by the weaker condition that it be separable has its uses for it permits us to deduce several representation theorems from Theorem 1 without much extra effort. 
We proceed now to do just that. First we formulate a result which gives us interesting examples of separable families of measurable transformations.

Proposition 1. Let $X$ be a separable metric space and let $@$ be the Borel $\sigma$-field of $X$. If $\mathcal{G}$ is

(a) a family of measurable transformations on $(X, \mathbb{Q})$ such that there is a countable family $\mathcal{J}_{0} \subseteq \mathcal{T}$ which is dense in $\mathcal{T}$ under pointwise convergence, or

(b) a locally compact second countable group acting measurably on $(X, \mathbb{Q})$, then $\mathcal{T}$ is separable.

We omit the proof, which is contained in the proofs of Corollaries 3 and 4 in Farrell [4]. It should be noted that Farrell assumes that $X$ is a Polish space, but his proofs of the separability of $\mathcal{T}$ in fact go through for separable metric $X$ without change.

Combining Theorem 1 with Proposition 1, we get

COROLlaRY 1. Let $X$ be a universally measurable separable metric space and let $\mathbb{Q}$ be the Borel $\sigma$-field of $X$. If $\sigma$ is as in (a) or (b) of Proposition 1, then every invariant measure is representable and has a unique representing measure.

This result includes Corollaries 3 and 4 of Farrell [4] and extends their validity from Polish spaces to universally measurable separable metric spaces. Also our result includes the main representation theorem of Varadarajan [16, Theorems 5.3 and 5.4] and extends its validity from analytic sets to universally measurable separable metric spaces.

The classical result of Kryloff and Bogoliouboff, quoted in the Introduction, also falls out of Theorem 1 . Indeed, if $\sigma$ is a family of continuous transformations on a compact metric space $X$ to $X$, then it is not difficult to see that there is a countable family $\mathscr{T}_{0} \subseteq \mathcal{T}$ such that $\mathscr{T}_{0}$ is dense in $\mathcal{T}$ under pointwise convergence (in fact, under uniform convergence), so, by Proposition $1, \mathcal{T}$ is separable. Hence Theorem 1 applies.

We conclude this section by deducing de Finetti's theorem on the representation of symmetric measures in a rather general context. Let $\left(X_{0}, \mathbb{Q}_{0}\right)$ be a measurable space and let $N$ be the set of positive integers. Let $X=X_{0}^{N}$ and denote by $\mathbb{Q}$ the product $\sigma$-field $\mathbb{Q}_{0} \times \mathbb{Q}_{0} \times \cdots$. Suppose $\mathcal{T}$ is the group of measurable transformations on $X$ which permute the coordinates of elements of $X$ and $\mathcal{T}_{0}$ is the subgroup of $\mathcal{T}$ consisting of transformations on $X$ which permute finitely many coordinates of elements of $X$. By a known result [7, Theorem 3.2], $\mathscr{P}_{\mathscr{T}}=\mathscr{P}_{\mathscr{T}_{0}}$ so that $\mathcal{T}$ is separable. Elements of $\mathscr{P}_{\text {g }}$ are called symmetric measures. Hewitt and Savage have proved that ex $\mathscr{P}_{\mathrm{g}}$ is just the set of product measures on $\mathscr{Q}$ having identical components [7, Theorem 5.3]. If, moreover, $\left(X_{0}, Q_{0}\right)$ is a perfect space, then, according to a result of Marczewski [11], so is $(X, \mathbb{Q})$. Therefore, with this assumption on $\left(X_{0}, \mathbb{Q}_{0}\right)$, Theorem 1 applies to $(X, \mathbb{Q}, \mathfrak{T})$ to yield 
Corollary 2. Let $\left(X_{0}, \mathscr{Q}_{0}\right)$ be a perfect space. Suppose $X=X_{0}^{N}$ and $\mathbb{Q}$ is the product $\sigma$-field on $X$, all of whose components are $\mathbb{Q}_{0}$. Then every symmetric measure on $\mathbb{Q}$ is a mixture of product measures on $\mathbb{Q}$ with identical components and the representing measure is unique.

In the terminology of Hewitt and Savage [7], we have proved above that if $\left(X_{0}, \mathbb{Q}_{0}\right)$ is a perfect space, then $\mathbb{Q}_{0}$ is presentable. It is easy to construct examples of perfect spaces which do not satisfy the hypotheses of the main representation theorem of Hewitt and Savage [7, Theorem 7.4]; consequently, our result cannot be deduced from theirs. On the other hand, nor can the Hewitt-Savage result be obtained from our Corollary 2. When the basic $\sigma$-field $\mathbb{Q}_{0}$ is countably generated, the above version of de Finetti's theorem is the most general known to the author. However, we do not know if the perfectness of the space $\left(X_{0}, Q_{0}\right)$ has anything to do with the presentability of $\mathbb{Q}_{0}$, though, of course, our methods do use the perfectness of $\left(X_{0}, \mathbb{Q}_{0}\right)$. The presentability of $\mathbb{Q}_{0}$ for countably generated $\mathbb{Q}_{0}$ seems to be an open problem.

5. The nonseparable case. We now turn our attention to the representation problem for invariant measures arising from the action of a not necessarily separable family of measurable transformations on a measurable space $(X, \mathbb{Q})$. Here the main difficulty is that the almost invariant $\sigma$-field $\bar{g}_{\text {g }}$ need not be sufficient for $\mathscr{P}_{\sigma}$. Below we describe two situations where invariant measures are representable even though $\mathcal{T}$ is not necessarily separable.

THEOREM 2. Suppose $(X, \mathbb{Q})$ is a measurable space and $\mathcal{T}$ is a compact group acting measurably on $(X, \mathbb{Q})$. Then every invariant measure is representable and has a unique representing measure.

Proof. As $\mathcal{T}$ is a compact group acting measurably on $(X, \mathbb{Q})$, it follows by a result of Farrell [4, Theorem 3] that the invariant $\sigma$-field $g_{\sigma}$ is sufficient for

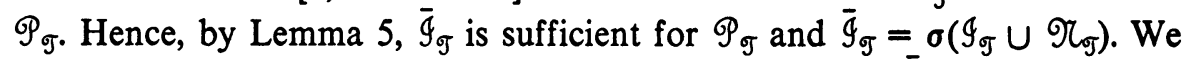
now verify that condition (b) of Lemma 1(ii) holds. Since $\bar{g}_{\sigma}=\sigma\left(g_{\sigma} \cup \vartheta_{\sigma g}\right)$, in order to show that condition (b) holds, it suffices to prove that if $\mu \in \mathscr{P}_{\mathfrak{g}}$, $A \in \mathscr{I}_{\text {g }}$ and $\mu(A)>0$, then there exists $\nu \in \operatorname{ex} \mathscr{P}_{\text {F }}$ such that $\nu(A)=1$. Let, then, $\mu \in \mathcal{P}_{\text {F }}, A \in \mathscr{I}_{\text {丁 }}$ and $\mu(A)>0$. Plainly, $A \neq \varnothing$. Let $x_{0} \in A$ and define $\theta$ on $Q$ by $\theta(E)=I_{E}\left(x_{0}\right)$. So $\theta$ is a $0-1$ valued measure on $Q$. Since the action of $\mathcal{T}$ on $(X, \mathbb{Q})$ is measurable and $\theta\left(T^{-1}(E)\right)=\int I_{E}(T(x)) d \theta(x)$ for every $T$ $\in \mathcal{T}$ and $E \in \mathbb{Q}$, it follows that, for fixed $E \in \mathbb{Q}$, the map $T \rightarrow \theta\left(T^{-1}(E)\right)$ is (Baire) measurable on $\mathcal{T}$. Consequently, we can define a measure $\nu$ on $\mathbb{Q}$ by $\nu(E)=\int \theta\left(T^{-1}(E)\right) d \lambda(T)$ where $\lambda$ is Haar measure on $\mathcal{T}$. It is easy to verify that $\nu \in \mathscr{P}_{\text {g }}$ and $\nu=\theta$ on $g_{\text {g. }}$. Hence $\nu \mid g_{\text {g }}$ is $0-1$ valued, and since $\bar{g}_{\text {g }}$

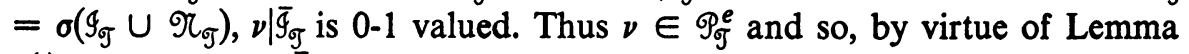

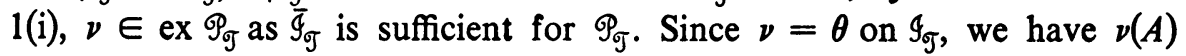


$=\theta(A)=I_{A}\left(x_{0}\right)=1$. So condition (b) of Lemma 1 (ii) is verified. Consequently, in view of the fact that $\bar{g}_{\text {g }}$ is sufficient for $\mathscr{P}_{\text {g }}$ and Lemma 1(ii), it follows that every invariant measure is representable and has a unique representing measure, which concludes the proof.

For the case of a finite group $\mathcal{G}$, the above result was proved by Hewitt and Savage [7, Theorem 11.7]. The next result, which extends the field of applicability of Theorem 1, is motivated by the proof of Theorem 2 .

Theorem 3. Let $(X, \mathbb{Q})$ be a perfect space and let $\mathcal{T}$ be a family of measurable transformations on $X$. Assume that either

(a) $\mathcal{I}_{\sigma}$ is sufficient for $\mathscr{P}_{\sigma}$, and there exist a countable family $\mathcal{F}_{0} \subseteq \mathcal{T}_{\text {and }} a$

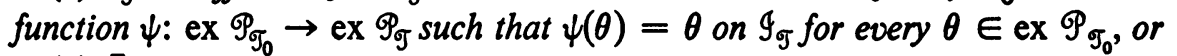

(b) $\overline{\mathcal{G}}_{\text {F }}$ is sufficient for $\mathscr{P}_{\mathrm{g}}$, and there exist a countable family $\mathcal{T}_{1} \subseteq \mathcal{T}$ and $a$ function $\psi_{1}:$ ex $\mathscr{P}_{\mathscr{T}_{1}} \rightarrow \operatorname{ex} \mathscr{P}_{\sigma_{F}}$ such that $\psi_{1}(\theta)=\theta$ on $\overline{\mathcal{G}}_{\text {g }}$ for every $\theta \in \operatorname{ex} \mathscr{P}_{\mathscr{F}_{1}}$.

Then every invariant measure is representable and has a unique representing measure.

Proof. Assume (a) holds. By Lemma 5, $\bar{g}_{\text {g }}$ is sufficient for $\Phi_{\sigma}$ and $\bar{g}_{\sigma}$ $=\sigma\left(g_{\sigma} \cup \vartheta_{\sigma}\right)$. We now check that condition (b) of Lemma 1(ii) holds. For this it suffices to check that if $\mu \in \mathscr{P}_{\text {g }}, A \in \mathscr{I}_{\text {g }}$ and $\mu(A)>0$, then there is $\nu \in \operatorname{ex} \mathscr{P}_{\text {g }}$ such that $\nu(A)=1$. This is because $\overline{\mathscr{I}}_{\text {g }}=\sigma\left(\Phi_{\text {g }} \cup \mathscr{T}_{\sigma}\right)$. So let $\mu \in \mathscr{P}_{\mathscr{T}}, A \in \mathscr{I}_{\mathscr{T}}$ and $\mu(A)>0$. Now $\mu \in \mathscr{P}_{\mathscr{T}_{0}}$; hence, by Theorem $1, \mu$ is representable, i.e., there is a measure $m$ on (ex $\left.\Phi_{\mathscr{T}_{0}}, \Sigma_{\sigma_{0}}\right)$ such that $\mu(E)$ $=\int_{\text {ex } \Phi_{g}} \theta(E) d m(\theta)$ for every $E \in \mathbb{Q}$. As $\mu(A)>0$, there is $\theta \in \operatorname{ex} \mathscr{P}_{\mathscr{T}_{0}}$ such that $\theta(A)>0$. Let $\nu=\psi(\theta)$. So $\nu \in$ ex $\mathscr{P}_{\mathscr{\sigma}}$. Since $A \in \mathscr{I}_{\text {F }}$ and $\psi(\theta)=\theta$ on $\mathscr{I}_{\mathscr{F}}$, it follows that $\nu(A)=\theta(A)>0$. As $\nu \in$ ex $\mathscr{P}_{\text {g }}$, in fact, $\nu(A)=1$ (Lemma 1(i)). Thus, condition (b) of Lemma 1(ii) is verified. Since $\bar{\Phi}_{\mathscr{\sigma}}$ is sufficient for $\mathcal{P}_{\sigma}$, Lemma 1(ii) yields the desired result.

The proof of the theorem in case (b) holds is similar and is omitted.

We conclude this section with the remark that we have been able to prove representation theorems for invariant measures only in the situations where $\bar{q}_{\mathrm{g}}$ is sufficient for $\mathscr{P}_{\sigma}$. We have no results for situations where this condition fails. Certainly our methods no longer work.

6. Examples. The examples that follow illustrate various aspects of the theory discussed in the previous sections. Some of the examples highlight the difficulties inherent in the situations where $\bar{g}_{\sigma}$ is not sufficient for $\mathscr{P}_{\text {g }}$.

EXAMPLE 1. Let $X_{0}=\{0,1\}$ and let $\mathbb{Q}_{0}$ be the discrete $\sigma$-field of $X_{0}$. Set $X=X_{0}^{N}$ and let $\mathcal{Q}$ be the product $\sigma$-field on $X$, all of whose components are $Q_{0}$. Suppose $\mathcal{T}$ is the group of transformations on $X$ which permute the coordinates of elements of $X$. The group $\mathcal{G}$ can be identified with the group of permutations of $N$ and so equipped with the topology inherited from $N^{N}$, where $N^{N}$ is equipped with the product of discrete topologies. So endowed 
with a topology, $\mathcal{T}$ becomes a Polish group acting measurably on $(X, \mathbb{Q})$. We have already observed in $\$ 4$ that $\sigma$ is separable. We now show that $g_{\sigma}$ is not sufficient for $\mathcal{P}_{\text {g. }}$. [But of course $\overline{\mathscr{G}}_{\mathscr{\sigma}}$ is sufficient for $\mathscr{P}_{\mathscr{\sigma}}$ for $\mathcal{T}$ is separable (Lemma 4).]

The structure of $g_{\sigma \sigma}$ is very simple. Indeed, $g_{\sigma}$ is atomic and has countably many atoms, all but one of which are countable. The set of all elements of $X$ for which infinitely many coordinates are 0 and infinitely many are 1 is the only uncountable atom of $9_{\text {g. }}$. For $p \in[0,1]$, denote by $\mu_{p}$ the product measure on $\mathbb{Q}$ with identical components, each component placing mass $p$ on 0 and mass $1-p$ on 1. Plainly, the measures $\mu_{p}, 0<p<1$, are continuous on $\mathbb{Q}$. It follows that the measures $\mu_{p}, 0<p<1$, agree on $9_{g}$. Consequently, $g_{g}$ is not separating for the family $\left\{\mu_{p}: 0<p<1\right\}$. Since $\left\{\mu_{p}: 0<p<1\right\} \subseteq \Phi_{\text {g }}$, it follows that $g_{\sigma}$ is not sufficient for $\mathcal{P}_{\text {g }}$.

The argument of the previous paragraph shows that any continuous measure $\mu \in \mathscr{P}_{\text {g }}$ is $0-1$ valued on $9_{\text {g. }}$. So, if we take $\mu$ to be a continuous mixture of the measures $\mu_{p}, 0<p<1$, for instance,

$$
\mu(E)=\int_{(0,1)} \mu_{p}(E) d \lambda(p)
$$

where $\lambda$ is Lebesgue measure and $E \in \mathbb{Q}$, we get an example of an invariant measure which is $0-1$ valued on $g_{g}$ but not ergodic.

ExAMPLE 2. This is taken from [1]. Let $X$ be the real line and $\mathcal{Q}$ the Borel $\sigma$ field of $X$. Let $\mathcal{G}$ be the family of all one-one transformations $T$ on $X$ onto $X$ such that $\{x \in X: T(x) \neq x\}$ is finite. Then $\mathcal{T}$ is a family of measurable transformations on $X$. It is easy to see that $\mathscr{P}_{\text {g }}$ is just the family of continuous measures on $\mathbb{Q}$. Moreover, $\bar{g}_{\mathscr{\sigma}}=\mathbb{Q}$ and hence sufficient for $\mathcal{P}_{\mathscr{\sigma}}$. We now show that there are no ergodic measures. For if $\mu \in \mathcal{P}_{\mathscr{\sigma}}^{e}$, then $\mu \mid \mathbb{Q}$ is $0-1$ valued. Since $\mathscr{Q}$ is countably generated and contains singletons, $\mu(\{x\})=1$ for some $x \in X$. But then $\mu$ is not continuous on $Q_{\text {, so }} \mu \notin \mathscr{P}_{\text {S }}$, which yields the desired contradiction. Thus, in the nonseparable situation, the perfectness of the space $(X, \mathbb{Q})$ and the sufficiency of $\bar{\Phi}_{\mathscr{F}}$ for $\mathscr{P}_{\mathscr{T}}$ need not ensure the representability of invariant measures.

Example 3. Let $(X, \mathbb{Q})$ be as in Example 2. We first introduce some terminology and notation. For $A \subseteq X$, let $-A=\{x \in X:-x \in A\}$. $\mathrm{A}$ measure $\mu$ on $\mathfrak{Q}$ is symmetric if $\mu(A)=\mu(-A)$ for every $A \in \mathbb{Q}$. If $x \in X, \delta(x)$ is the measure on $\mathbb{Q}$ which concentrates on the point $x$.

For $a>0$, let $T_{a}$ be the transformation on $X$ to $X$ which interchanges the points $a$ and $-a$ and leaves the other points fixed. Let $\mathcal{T}=\left\{T_{a}: a>0\right\}$, so $\mathcal{T}$ is a family of measurable transformations on $X$. The following facts are easy to verify: 
(i) For a measure $\mu$ on $\mathbb{Q}_{,} \mu \in \mathscr{P}_{\mathscr{F}}$ if and only if $\mu(\{a\})=\mu(\{-a\})$ for every $a>0$, so that, in particular, all continuous measures on $\mathscr{Q}$ are invariant;

(ii) $\bar{g}_{\text {g }}=\{A \in \mathbb{Q}: A=-A\}$;

(iii) the extreme points of $\mathscr{P}_{\mathscr{\sigma}}$ are just the measures $\frac{1}{2} \delta(a)+\frac{1}{2} \delta(-a), a \geqslant 0$ (use the fact that $\bar{\Phi}_{\mathscr{F}}$ is countably generated and has atoms of the form $\{a,-a\}$ to verify this). It is evident from this that condition (b) of Lemma 1(ii) holds. Now, if $\mu \in \mathscr{P}_{\text {g }}$ and $\mu$ is representable, it is clear that $\mu$ is symmetric. Taking $\mu$ to be a continuous measure on $\mathbb{Q}$ such that $\mu((-\infty, 0)) \neq \mu((0, \infty))$, we see that $\mu \in \mathscr{P}_{\mathscr{\sigma}}$ but $\mu$ is not representable. Consequently, in general, condition (b) of Lemma 1(ii) is not a sufficient condition for the representability of invariant measures.

EXAMPLE 4. This example shows that ergodic measures need not be extreme points. Let $(X, \mathbb{Q})$ be as in Example 2. We need the following lemma due to B. V. Rao [14]; see [14] for a proof.

LEMMA 8. There is a one-one function $f$ on $X$ onto $X$ such that (i) $f=f^{-1}$, and (ii) for every set $E \in \mathbb{Q}$ such that both $E$ and $E^{c}$ are uncountable, $f^{-1}(E) \notin \mathbb{Q}$.

Let $f: X \rightarrow X$ be as in the above lemma. For each $x \in X$, let $T_{x}$ be the transformation of $X$ to $X$ which interchanges the points $x$ and $f(x)$ and leaves the other points fixed. Let $\mathcal{T}=\left\{T_{x}: x \in X\right\}$, so $\mathcal{T}$ is a family of measurable transformations on $X$. Plainly, a measure $\mu$ is invariant if and only if $\mu(\{x\})=\mu(\{f(x)\})$ for every $x \in X$. Consequently, every continuous measure is invariant. It is not difficult to see that $\bar{\Phi}_{\sigma}=\left\{A \in \mathbb{Q}: f^{-1}(A)=A\right\}$. We now show that every continuous measure on $\mathscr{Q}$ is ergodic, but that no continuous measure on $\mathbb{Q}$ is an extreme point of $\mathscr{P}_{\text {}}$. Let, then, $\mu$ be a continuous measure on $\mathcal{Q}$. If $A \in \bar{\Phi}_{\mathscr{T}}$, then $A \in \mathcal{Q}$ and $f^{-1}(A)=A$, so that, by Lemma $8, A$ is either countable or cocountable. So, for every $A \in \bar{g}_{\text {g }}, \mu(A)=0$ or 1 , as $\mu$ is continuous. This proves $\mu$ is ergodic. Since $\mu$ is continuous on $\mathbb{Q}$ and $\mathscr{Q}$ is countably generated, $\mu$ is nonatomic on $\mathbb{Q}$. Choose a set $B \in \mathbb{Q}$ such that $\mu(B)=\frac{1}{2}$. Since for any $T \in \mathcal{T}, B \triangle T^{-1}(B)$ contains at most two points, it follows that $\mu\left(B \triangle T^{-1}(B)\right)=0$ for every $T \in \mathcal{T}$. Define measures $\lambda_{1}, \lambda_{2}$ on $\mathbb{Q}$ by $\lambda_{1}(E)=\mu(B \cap E) / \mu(B), \lambda_{2}(E)=\mu\left(B^{c} \cap E\right) / \mu\left(B^{c}\right)$. Using the fact that $B$ is $\mu$-almost invariant, one checks that $\lambda_{1}, \lambda_{2} \in \mathscr{P}_{\mathscr{\sigma}}$. Plainly, $\lambda_{1}$ $\neq \lambda_{2}$ and $\mu=\frac{1}{2} \lambda_{1}+\frac{1}{2} \lambda_{2}$. Hence $\mu$ is not an extreme point of $\mathscr{P}_{\text {g }}$.

EXAMple 5. Let $(X, Q)$ be as in Example 2. According to a classical result (see, for example, [10, pp. 525, 532]), there is an uncoutable set $M \subseteq(0, \infty)$ such that $M$ is universally null, i.e., for every continuous measure $\mu$ on $\mathbb{Q}$, $\mu^{*}(M)=0$ (here $\mu^{*}$ is the outer measure induced by $\mu$ ). The continuum hypothesis is needed to prove the existence of such a set. Let $N=M \cup-M$, so that $N$ is uncountable, universally null, does not contain 0 , and $N=-N$. 
For $x \in M$, let $T_{x}$ be the transformation of $X$ to $X$ which interchanges $x$ and $-x$ and leaves the other points fixed. Let $\mathcal{T}=\left\{T_{x}: x \in M\right\}$, so $\mathcal{T}$ is a family of measurable transformations on $X$. Clearly, a measure $\mu$ on $\mathcal{Q}$ is invariant if and only if $\mu(\{x\})=\mu(\{-x\})$ for every $x \in M$. It is not difficult to verify that

$$
\bar{g}_{\text {ब }}=\left\{A \cup A_{0}: A \in \mathbb{Q}, A_{0} \in \mathbb{Q}, A=-A \text { and } A_{0} \subseteq N^{c}\right\} \text {. }
$$

Burkholder has proved that $\bar{\Phi}_{\sigma}$ is not sufficient for the family of symmetric measures on $\mathbb{Q}$; see [3, p. 1192]. As every symmetric measure is invariant, this proves that $\bar{\Phi}_{\mathscr{T}}$ is not sufficient for $\mathscr{P}_{\mathscr{T}}$.

We now show that every $\mu \in \mathscr{P}_{\text {F }}$ is representable and has a unique representing measure. First note that

$$
\text { ex } \mathscr{P}_{\mathscr{T}}=\left\{\frac{1}{2} \delta(x)+\frac{1}{2} \delta(-x): x \in M\right\} \cup\left\{\delta(x): x \in N^{c}\right\} .
$$

Consider now the function $\varphi: X \rightarrow$ ex $\mathscr{P}_{\mathscr{\sigma}}$ defined by $\varphi(x)=\frac{1}{2} \delta(x)+\frac{1}{2} \delta(-x)$ if $x \in N$, and $\varphi(x)=\delta(x)$, if $x \in N^{c}$. Check that $\varphi$ is a $\sigma$-isomorphism of the $\sigma$-fields $\sigma\left(\overline{\mathscr{G}}_{\text {g }} \cup\{N\}\right)$ and $\Sigma_{\sigma}$. If $\mu \in \mathscr{P}_{\text {g }}$ and $\mu$ is discrete, then it is obvious that $\mu$ is representable and has a unique representing measure. So, then, let $\mu$ be a continuous measure. As $N$ is universally null, $N$ is $\mu$-measurable. Consequently, there is a unique countably additive extension $\mu$ of $\mu$ to $\sigma(\mathbb{Q} \cup\{N\})$. Moreover, $\mu(N)=0$. Now, for any $E \in \mathbb{Q}$, we have

$$
\begin{aligned}
\mu(E) & =\bar{\mu}\left(E \cap N^{c}\right)=\int_{N^{c}} \varphi(x)(E) d \bar{\mu}(x)=\int_{X} \varphi(x)(E) d \bar{\mu}(x) \\
& =\int_{\mathrm{ex} \Phi_{g}} \nu(E) d \mu \varphi^{-1}(\nu),
\end{aligned}
$$

so that $\mu \varphi^{-1}$ is a representing measure for $\mu$. Suppose now that $m_{1}, m_{2}$ are representing measures for $\mu$. Use the $\sigma$-isomorphism $\varphi$ to transfer the measures $m_{i}$ on $\Sigma_{\mathscr{\sigma}}$ to measures $\lambda_{i}$ on $\sigma\left(\bar{\Phi}_{\mathscr{G}} \cup\{N\}\right), i=1,2$. Then, for every $E \in \mathbb{Q}$, we have

$$
\mu(E)=\int_{X} \varphi(x)(E) d \lambda_{1}(x)=\int_{X} \varphi(x)(E) d \lambda_{2}(x) .
$$

It follows from this that $\lambda_{1}=\mu=\lambda_{2}$ on $\bar{\Phi}_{\text {g }}$. As $N$ is universally null, there is a set $B \in \mathbb{Q}$ such that $B \subseteq N^{c}$ and $\mu(B)=1$. Note that then $B$ $\in \bar{g}_{\text {g }}$ and so $\lambda_{1}(B)=\lambda_{2}(B)=1$. Now let $F \in \sigma\left(\left(\bar{G}_{\text {g }} \cup\{N\}\right)\right.$, so $F=\left(E_{1}\right.$ $\cap N) \cup\left(E_{2} \cap N^{c}\right)$, where $E_{i} \in \bar{\Phi}_{\sigma}, i=1$, 2. Then

$$
\lambda_{i}(F)=\lambda_{i}(F \cap B)=\lambda_{i}\left(E_{2} \cap B\right)=\lambda_{i}\left(E_{2}\right), \quad i=1,2 .
$$

As $E_{2} \in \bar{\Phi}_{\text {g }}, \lambda_{1}\left(E_{2}\right)=\lambda_{2}\left(E_{2}\right)$, so

$$
\lambda_{1}(F)=\lambda_{2}(F) .
$$


Thus, $\lambda_{1}=\lambda_{2}$ on $\sigma\left(\overline{\mathscr{T}}_{\text {Fु }} \cup\{N\}\right)$, so $m_{1}=m_{2}$ on $\Sigma_{\text {T. }}$. So $\mu$ is representable and has a unique representing measure. Finally, if $\mu \in \mathscr{P}_{\text {g }}$ is arbitrary, we can decompose $\mu$ into its discrete and continuous parts, which will be again invariant, and then we can use the results established above for discrete and continuous invariant measures to show that $\mu$ is representable and has a unique representing measure.

Thus, the sufficiency of $\bar{g}_{\mathscr{T}}$ for $\mathscr{P}_{\mathscr{T}}$ is not a necessary condition for the representability of invariant measures. Note that in proving Lemma 1(ii) one uses the fact that the map $A \rightarrow\left\{\nu \in \operatorname{ex} \mathscr{P}_{\mathscr{\sigma}}: \nu(A)=1\right\}$ is a $\sigma$-homomorphism of $\bar{g}_{\sigma}$ onto $\Sigma_{\sigma}$. This is a consequence of the sufficiency of $\bar{g}_{\sigma}$ for $\Phi_{\sigma}$. In the present example, it is clear that the above map is not a $\sigma$-homomorphism of $\bar{G}_{g}$ onto $\Sigma_{\sigma}$. Nonetheless, every invariant measure is representable. This suggests that, when $\bar{\Phi}_{\sigma}$ is not sufficient for $\mathscr{P}_{\mathscr{T}}$ but, in some sense, there are enough extreme points, we should look for a larger $\sigma$-field, which may not be contained in $\mathbb{Q}$, to play the role of $\overline{\mathscr{G}}_{\mathrm{G}}$. This will involve us in the existence of extensions of invariant measures. We have not yet explored this.

EXAMple 6. Let $(X, \mathbb{Q})$ be as in the previous example. Using a result of $\mathrm{F}$. Bernstein (see, for example, [10, p. 514]), we can get a set $N \subseteq X$ such that $N=-N, 0 \notin N$, and neither $N$ nor $N^{c}$ contains an uncountable Borel subset of $X$. As in Example 5, for each $x \in N \cap(0, \infty)$, let $T_{x}$ be the transformation of $X$ to $X$ which interchanges $x$ and $-x$ and leaves the other points fixed. Let $\mathscr{T}=\left\{T_{x}: x \in N \cap(0, \infty)\right\}$. Then $\mathscr{P}_{\mathscr{T}}, \bar{g}_{\mathscr{T}}$, ex $\mathscr{P}_{\mathscr{T}}$ and $\Sigma_{\mathscr{T}}$ can be described just as in Example 5. If $\varphi$ is as in Example 5, then one shows that $\varphi$ is a $\sigma$ isomorphism of the $\sigma$-fields $\sigma\left(\bar{\Phi}_{\mathscr{G}} \cup\{N\}\right)$ and $\Sigma_{\mathscr{g}}$. Using this one shows that every $\mu \in \mathcal{P}_{\mathscr{G}}$ is representable. For instance, if $\mu$ is a continuous measure on $Q$, then we obtain a countably additive extension $\mu$ of $\mu$ by setting $\bar{\mu}(E)$ $=\mu^{*}\left(E \cap N^{c}\right)$ for $E \in \sigma(\mathscr{Q} \cup\{N\})$. Since $\mu^{*}\left(N^{c}\right)=1, \bar{\mu}\left(N^{c}\right)=1$. Now a computation as in Example 5 shows that $\mu \varphi^{-1}$ is a representing measure for $\mu$.

Now let $\mu$ be a continuous symmetric measure on $Q$, so $\mu \in \mathcal{P}_{\mathcal{T}}$. We show that $\mu$ has at least two distinct representing measures. Define countably additive extensions $\mu_{1}, \mu_{2}$ of $\mu$ to $\sigma(\mathscr{Q} \cup\{N\})$ as follows:

$$
\mu_{1}(E)=\mu^{*}\left(E \cap N^{c}\right) \text { and } \mu_{2}(E)=\mu^{*}(E \cap N) .
$$

It is easy to check that $\mu_{1} \varphi^{-1}$ and $\mu_{2} \varphi^{-1}$ are representing measures for $\mu$ (one uses the fact that $\mu$ is symmetric to check that $\mu_{2} \varphi^{-1}$ is a representing measure for $\mu$ ). But $\mu_{1} \neq \mu_{2}$ on $\sigma\left(\bar{\Theta}_{\text {g }} \cup\{N\}\right)$ for $\mu_{1}(N)=0$ and $\mu_{2}(N)=1$. So $\mu_{1} \varphi^{-1}$ and $\mu_{2} \varphi^{-1}$ are distinct measures on $\Sigma_{\sigma}$.

7. Acknowledgements. I am greatly indebted to D. Basu and B. V. Rao for many helpful conversations on this and related topics. Example 3 was suggested to me by D. Basu and I received much help in the construction of 
Example 4 from B. V. Rao. I am grateful to the referee for several helpful suggestions.

\section{REFERENCES}

1. D. Basu, On sufficiency and invariance, Essays in Probability and Statistics (S. N. Roy Memorial Volume, R. C. Bose et al., editors), Univ. of North Carolina Press, Chapel Hill, N. C., 1970. MR 42 \# 1253.

2. J. R. Blum and D. L. Hanson, On invariant probability measures. I, Pacific J. Math. 10 (1960), 1125-1129. MR 24 \#A3260.

3. D. L. Burkholder, Sufficiency in the undominated case, Ann. Math. Statist. 32 (1961), 1191-1200. MR 24 \#A1139.

4. R. H. Farrell, Representation of invariant measures, Illinois J. Math. 6 (1962), 447-467. MR 27 \#265.

5. D. A. Freedman and L. E. Dubins, Measurable sets of measures, Pacific J. Math. 14 (1964), 1211-1222. MR 30 \#4887.

6. D. Freedman, Markov chains, Holden-Day, San Francisco, Calif., 1971. MR 45 \# 1263.

7. E. Hewitt and L. J. Savage, Symmetric measures on Cartesian products, Trans. Amer. Math. Soc. 80 (1955), 470-501. MR 17, 863.

8. M. Jirina, Conditional probabilities on o-algebras with countable basis, Czechoslovak. Math. J. 4(79) (1954), 372-380; English transl., Selected Transl. Math. Stat. and Prob., vol. 2, Amer. Math. Soc., Providence, R. I., 1962, pp. 79-86. MR 16, 1034; 27 \#812.

9. N. Kryloff and N. Bogoliouboff, La théorie générale de la mesure dans son application a l'étude des systèmes dynamiques de la mécanique non linéaire, Ann of Math. (2) 38 (1937), 65-113.

10. K. Kuratowski, Topology. Vol. 1, 5th ed., PWN, Warsaw; Academic Press, New York; "Mir", Moscow, 1966. MR 36 \#839, \#840.

11. E. Marczewski, On compact measures, Fund. Math. 40 (1953), 113-124. MR 15, 610.

12. J. C. Oxtoby, Ergodic sets, Bull. Amer. Math. Soc. 58 (1952), 116-136, 850.

13. R. R. Phelps, Lectures on Choquet's theorem, Math. Studies, no. 7, Van Nostrand, Princeton, N. J., 1966. MR 33 \# 1690.

14. B. V. Rao, On Borel structures, Colloq. Math. 21 (1970), 199-204.

15. C. Ryll-Nardzewski, On quasi-compact measures, Fund. Math. 40 (1953), 125-130. MR 5, 610.

16. V. S. Varadarajan, Groups of automorphims of Borel spaces, Trans. Amer. Math. Soc. 109 (1963), 191-220. MR 28 \#3139.

Department of Mathematics, Indian Statistical Institute, Calcutta 700 035, India 